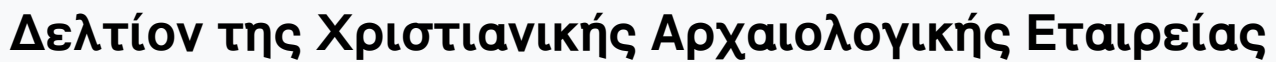

Tó 21 (2000)

$\Delta \varepsilon \lambda$ tíov XAE 21 (2000), Пврíoঠoc $\Delta^{\prime}$

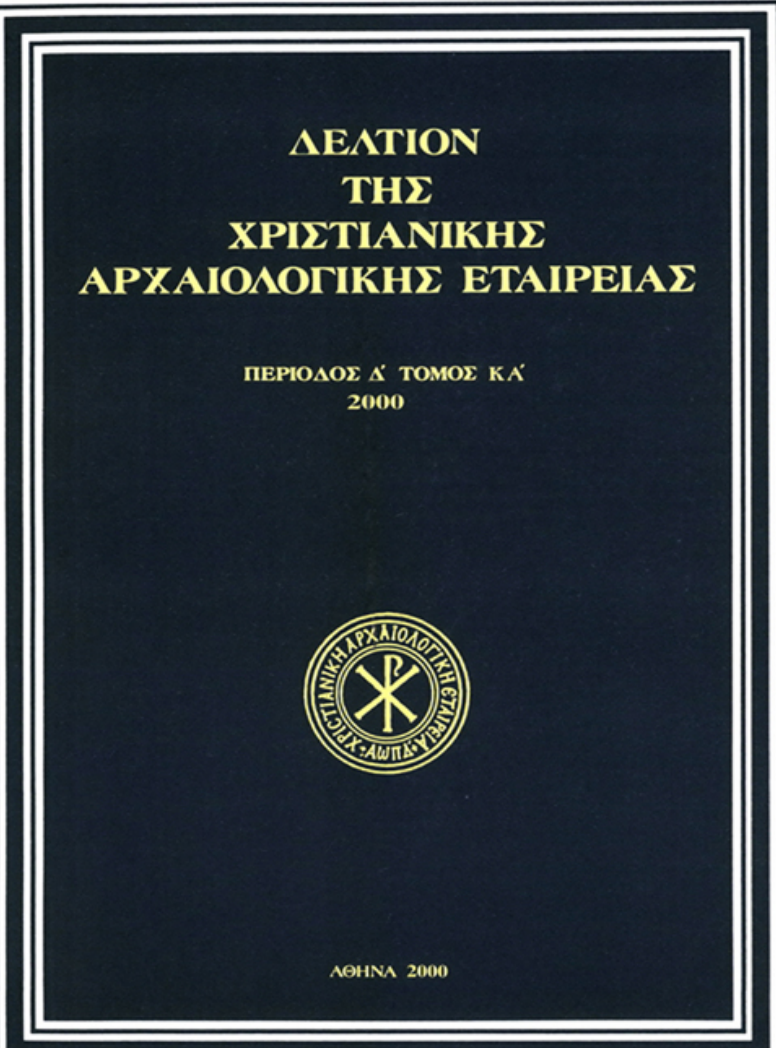

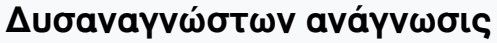

KWvotavtívos $\Sigma K A M \Pi A B I A \Sigma$

doi: $10.12681 /$ dchae.562

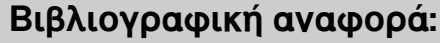

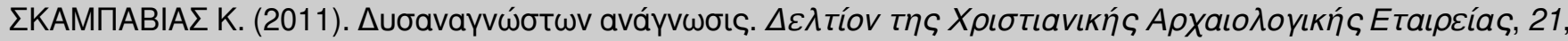
241-248. https://doi.org/10.12681/dchae.562 


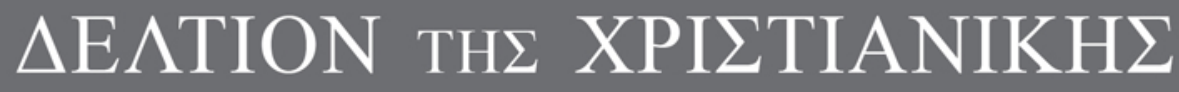 APXAIO $\Lambda$ OГIKH $\Sigma$ ETAIPEIA $\Sigma$}

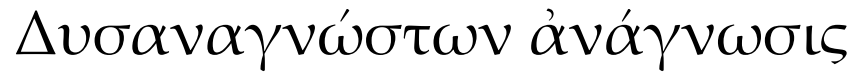

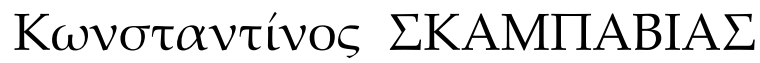

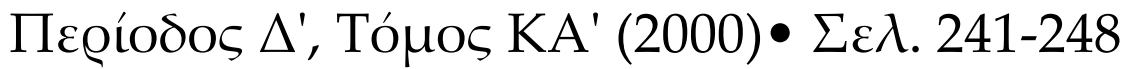
A@HNA 2000 


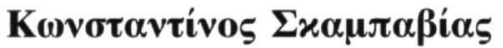

\section{$\Delta \mathrm{Y} \Sigma \mathrm{ANA} \Gamma \mathrm{N} \Omega \Sigma \mathrm{T} \Omega \mathrm{N}$ ANA $\mathrm{NN} \Omega \Sigma \mathrm{I} \Sigma$}

\section{$\Sigma$}

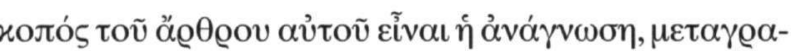

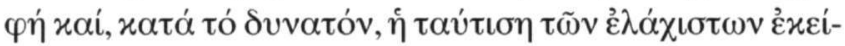

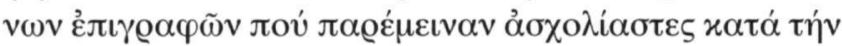

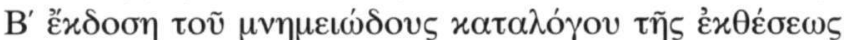

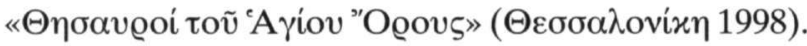

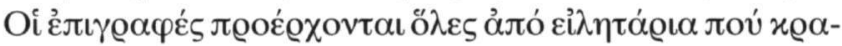

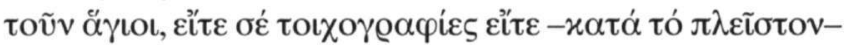

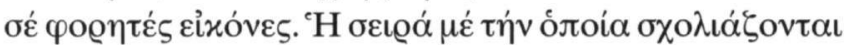

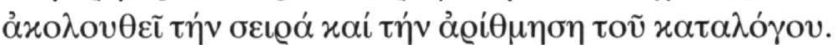

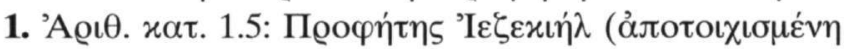

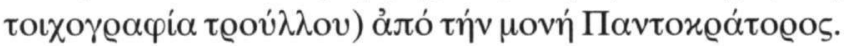

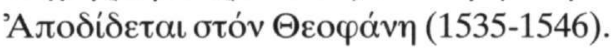

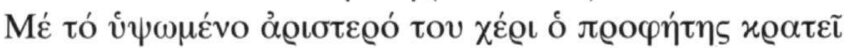

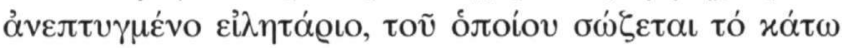

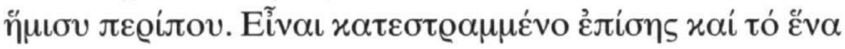

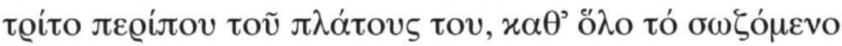
$\mu \tilde{\eta}$ นоร.

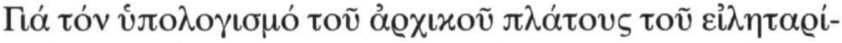

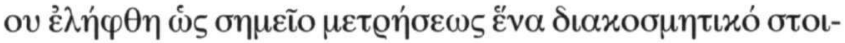

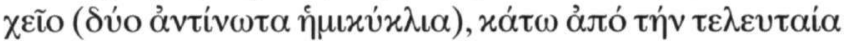

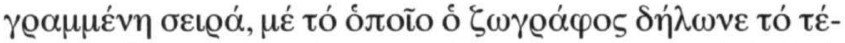

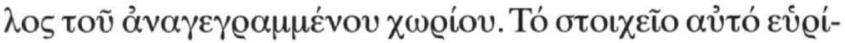

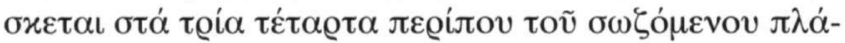

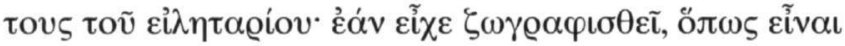

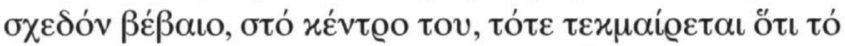

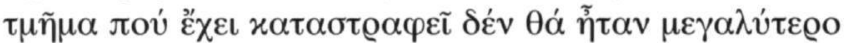

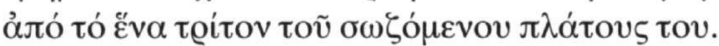

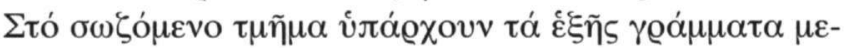

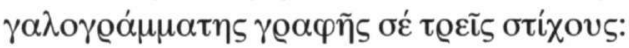

$$
\begin{aligned}
& { }^{\prime} E I ![\ldots \\
& K(Y P I O) C \cdot K(A I)[\ldots
\end{aligned}
$$$$
K E ́ \text { ME [... }
$$

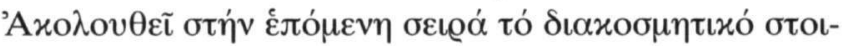

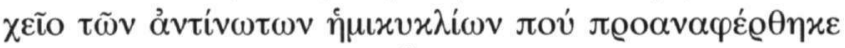

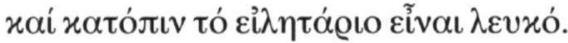

'A

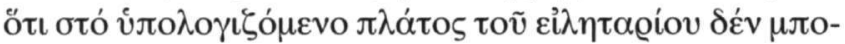

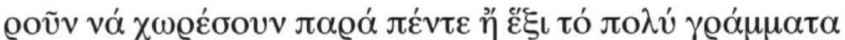

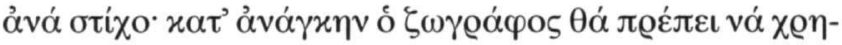

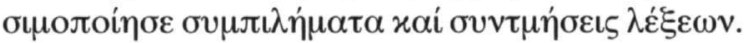

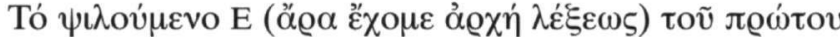

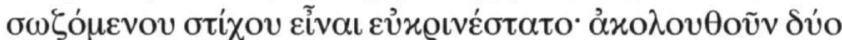

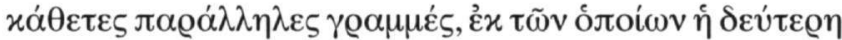

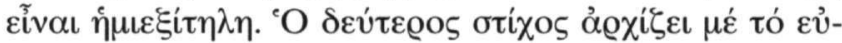

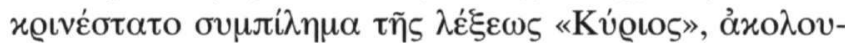

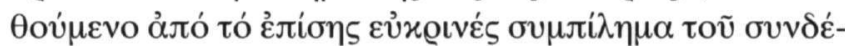

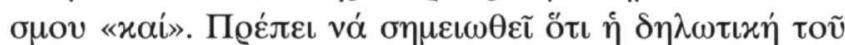

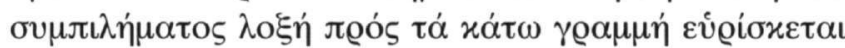

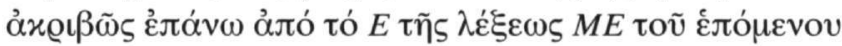

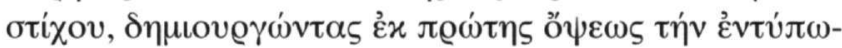

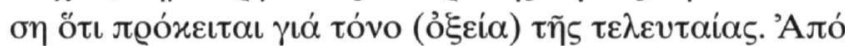

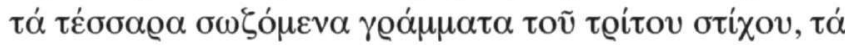

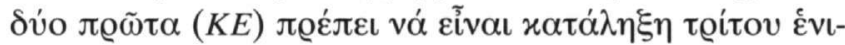

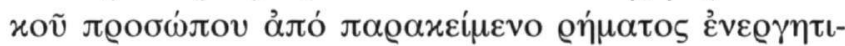

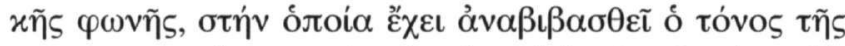

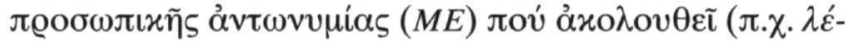
$\lambda v x \varepsilon \dot{\varepsilon} \mu)$.

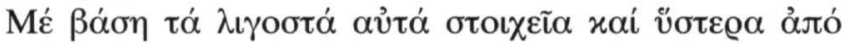

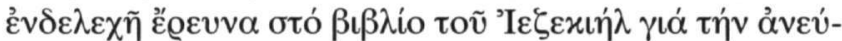

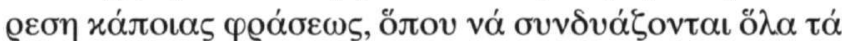

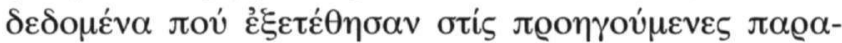

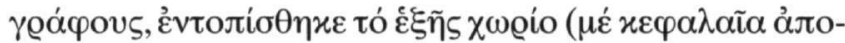

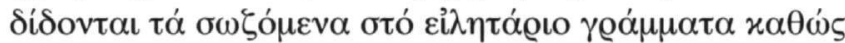

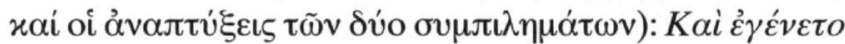

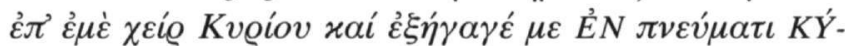

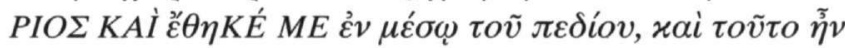

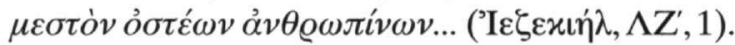

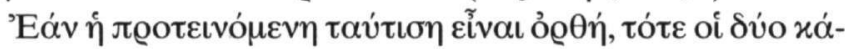

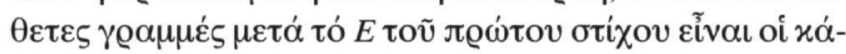

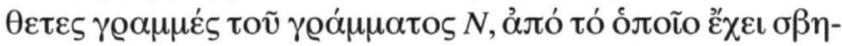

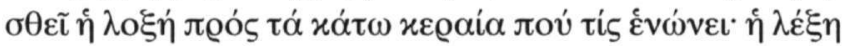

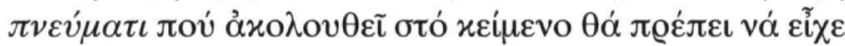

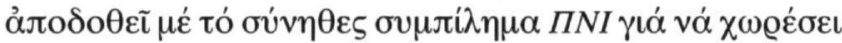

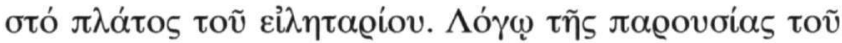

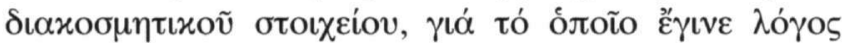

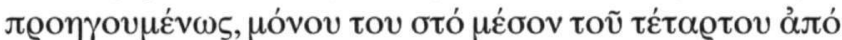

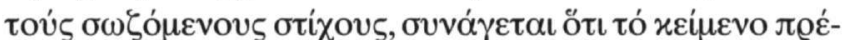

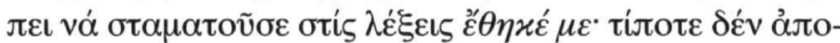

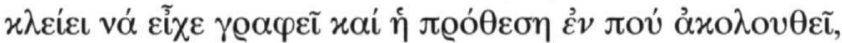

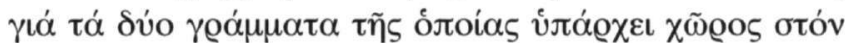




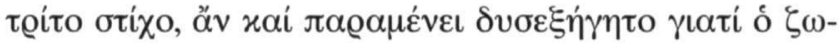

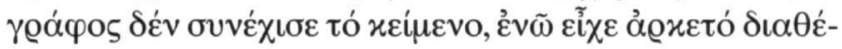

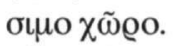

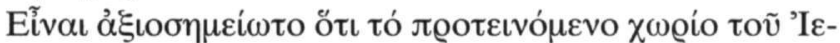

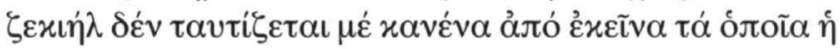

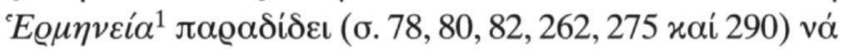

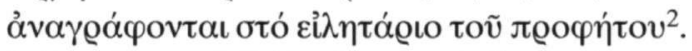

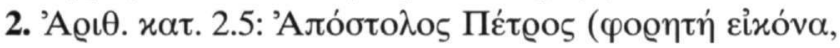

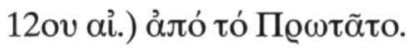

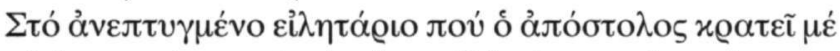

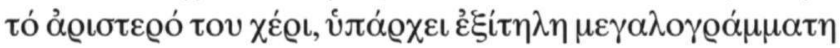

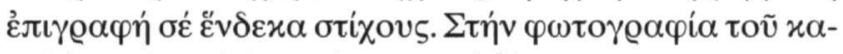

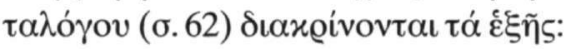

$$
\begin{aligned}
& +A[\ldots . . .] \text { OI } A \Pi \Pi O \\
& \text { QEMMENOI İ ITCAN } \\
& \text { KATKIAN K(AI) } \triangle O \perp Q Q N K(A I) \\
& \Phi \Theta O N[. . .] \text { ATTA } A A \Lambda I A N
\end{aligned}
$$

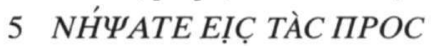

$$
\begin{aligned}
& \text { EYXА̀C O ГАР КАTAАA }
\end{aligned}
$$

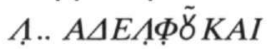

$$
\begin{aligned}
& \text { KPINQNSTONA } \\
& \triangle E \Lambda \Phi O N A Y T O \tilde{Y} \\
& 10 \text { MENEIENTS } \\
& \Theta A N A T \Omega+
\end{aligned}
$$

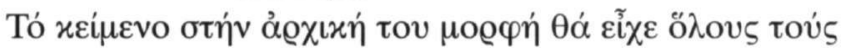

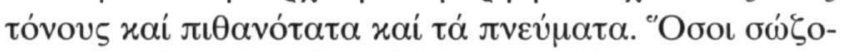

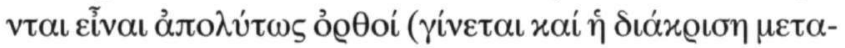

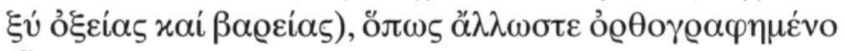

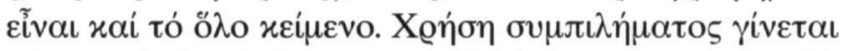

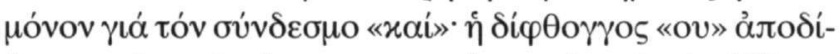

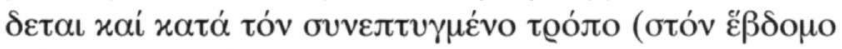

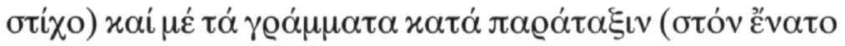

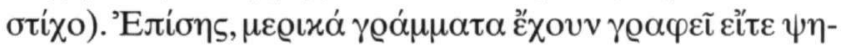

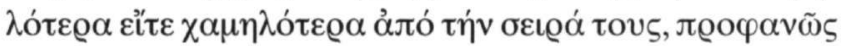

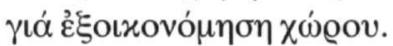

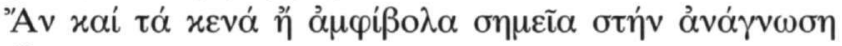

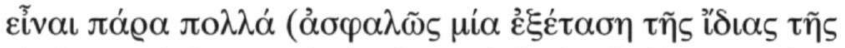

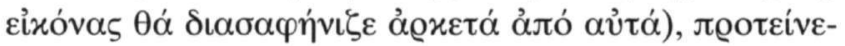

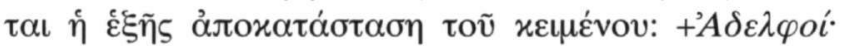

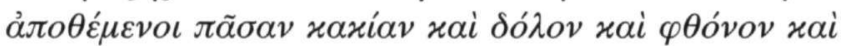

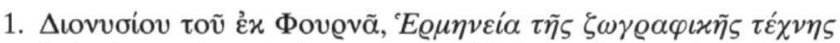

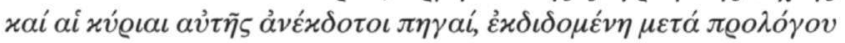

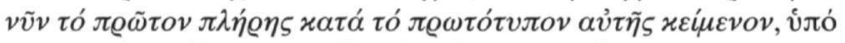

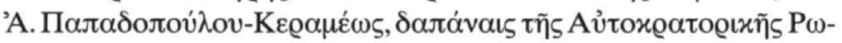

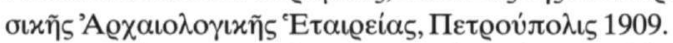

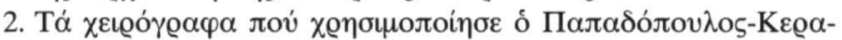

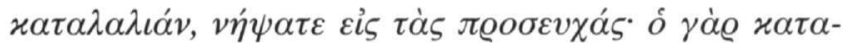

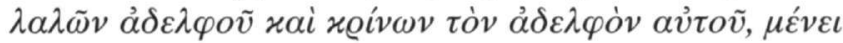
$\dot{\varepsilon} v \tau \tilde{\omega} \theta \alpha v \alpha ́ \tau \omega+$.

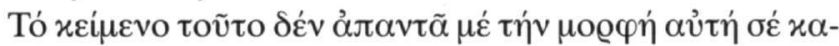

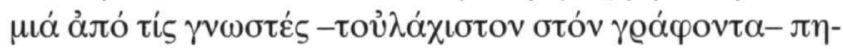

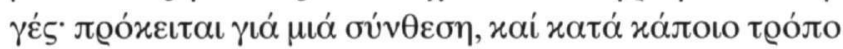

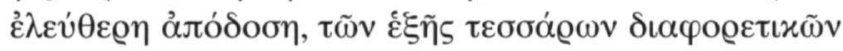

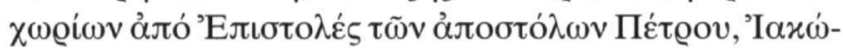

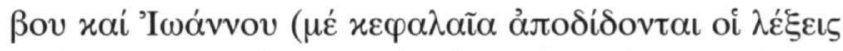

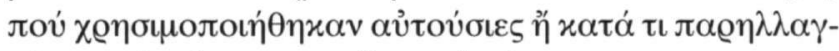
$\mu \varepsilon \dot{v \varepsilon \varsigma ~ \sigma \tau o ́ ~ x \varepsilon i ́ \mu \varepsilon v o ~ \tau o v ̃ ~ \varepsilon i ̉ ~ \eta \tau \alpha \varrho i ́ o v): ~}$

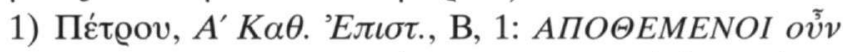

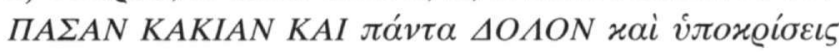

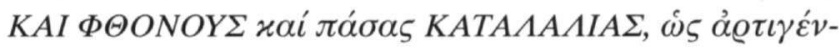

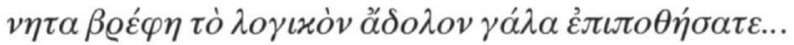

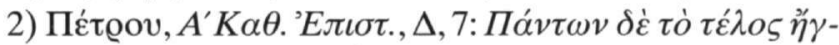

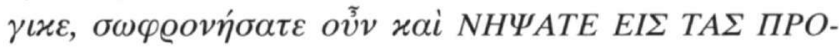
$\Sigma E Y X A \Sigma$.

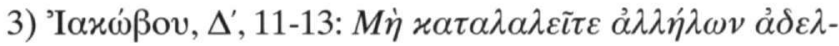

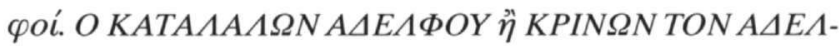

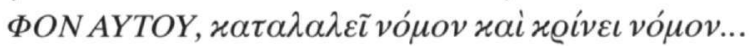

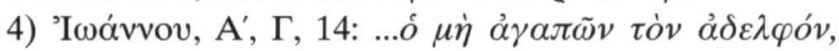
MENEI ENTS $\Theta A N A T \Omega$.

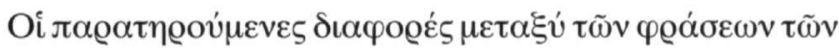

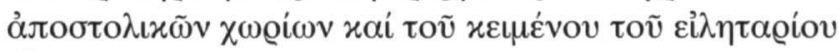

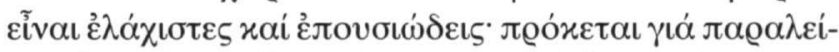

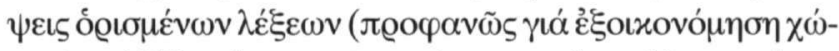

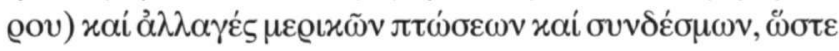

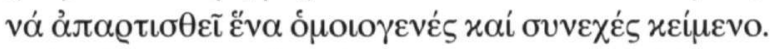

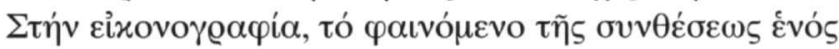

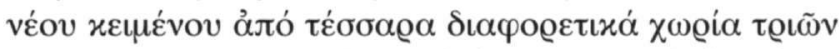

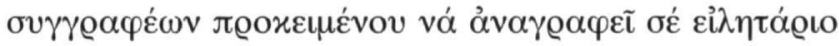

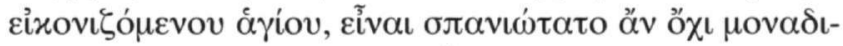

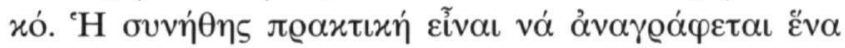

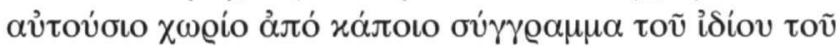

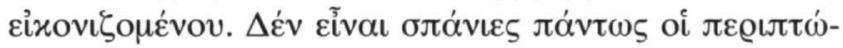

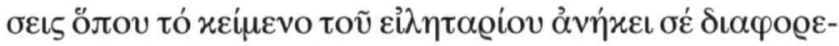

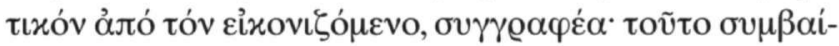

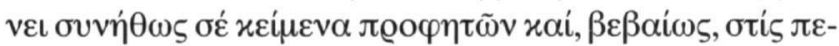

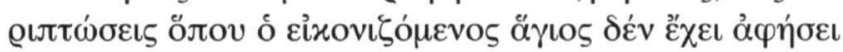

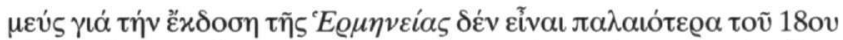

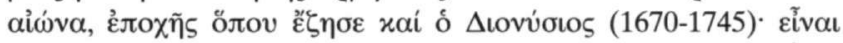

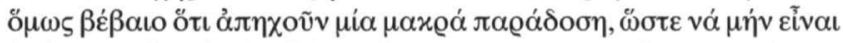

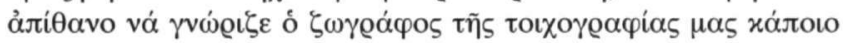

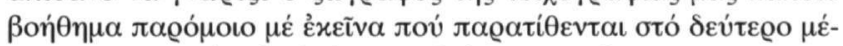

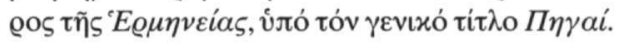




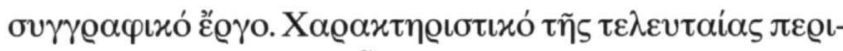

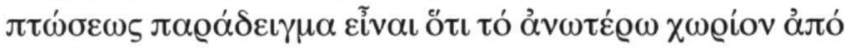

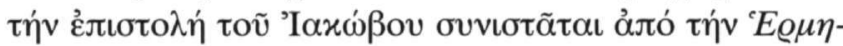

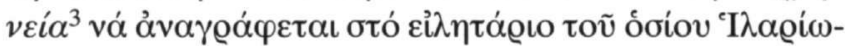

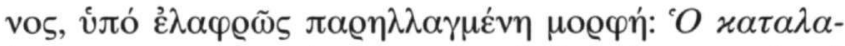

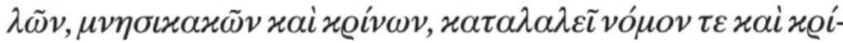

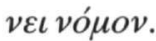

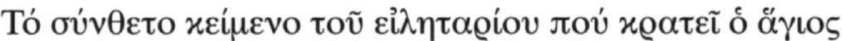

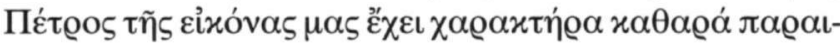

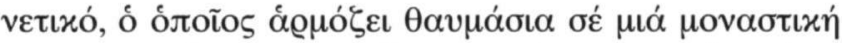

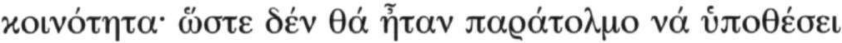

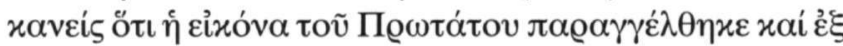

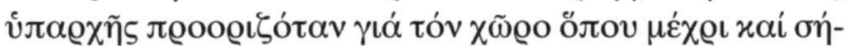
$\mu \varepsilon \varrho \alpha \varphi v \lambda \alpha \dot{\sigma} \sigma \sigma \varepsilon \tau \alpha$.

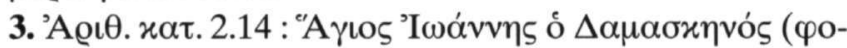

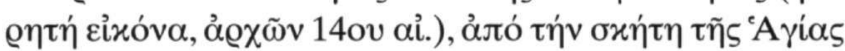
"Avvฑร.

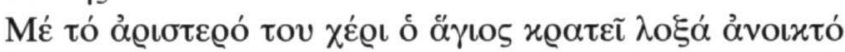

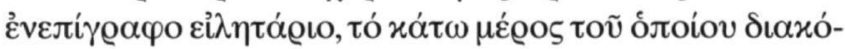

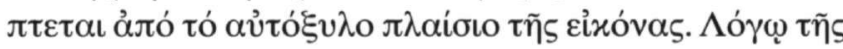

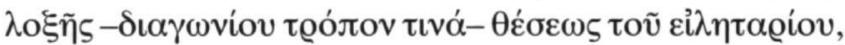

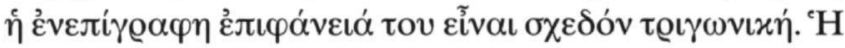

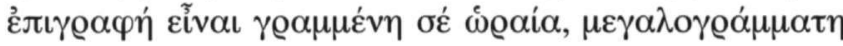

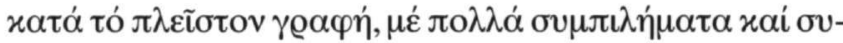

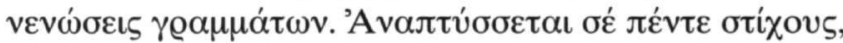

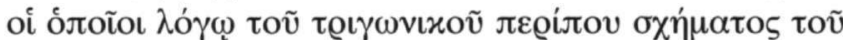

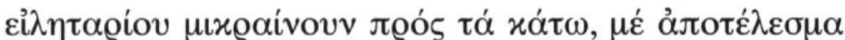

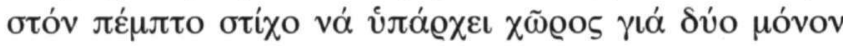

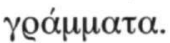

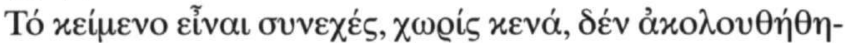

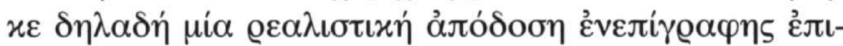

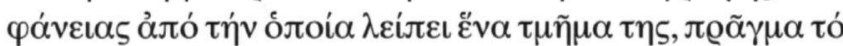

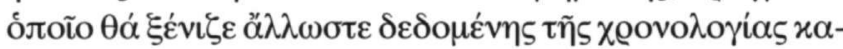

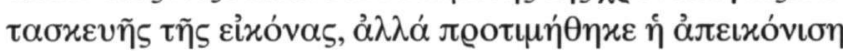

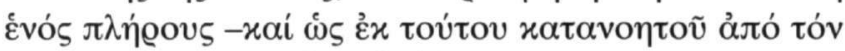

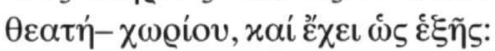

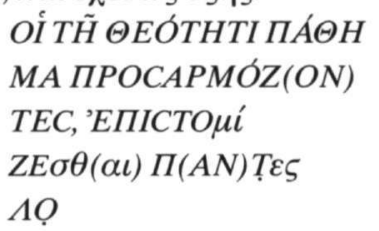

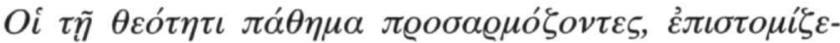
$\sigma \theta \alpha \iota \pi \dot{\alpha} \nu \tau \varepsilon \zeta \Lambda O[\Gamma \Omega]$ ทे $\Lambda E[\Gamma \Omega]$.

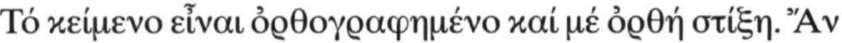

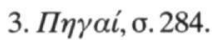

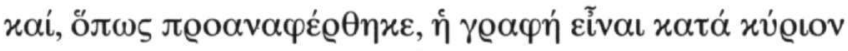

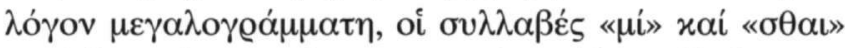

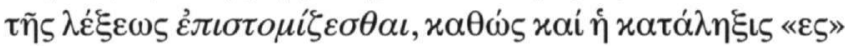

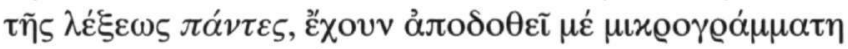

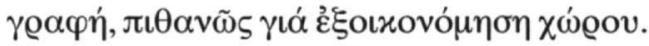

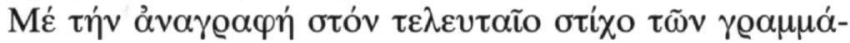

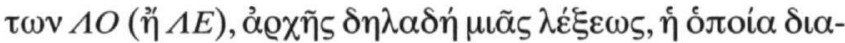

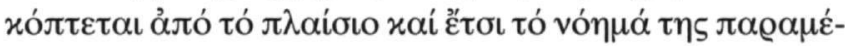

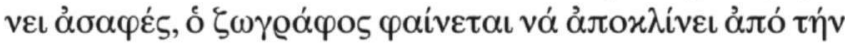

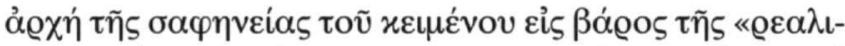

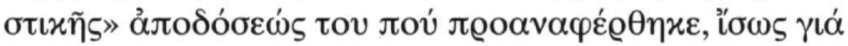

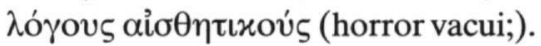

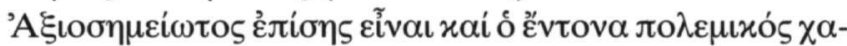

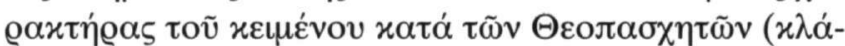

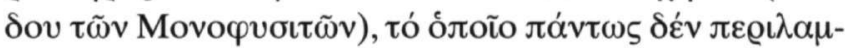

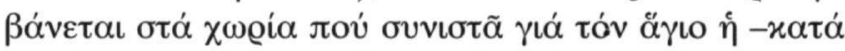

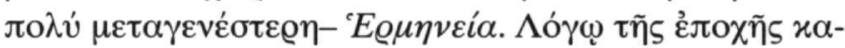

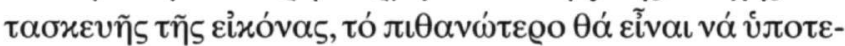

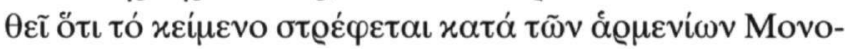

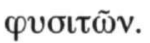

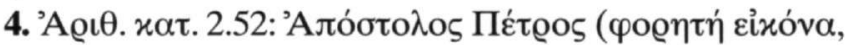

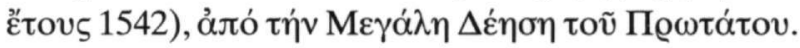

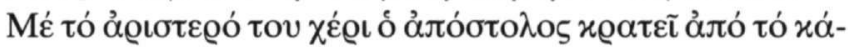

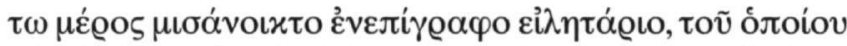

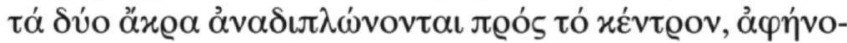

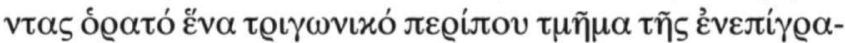

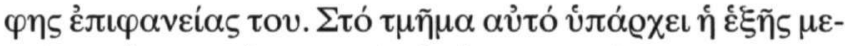

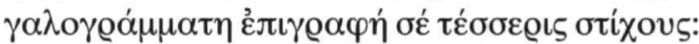

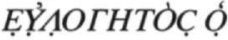

$$
\begin{aligned}
& \Theta(E O) C K(A I) \\
& \Pi(A T) H P \\
& \text { TOY K ГYPIOY... }
\end{aligned}
$$

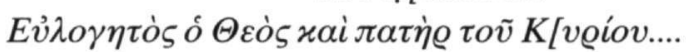

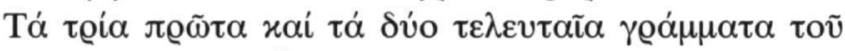

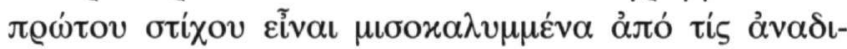

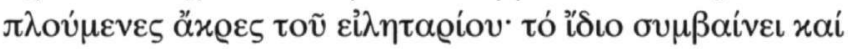

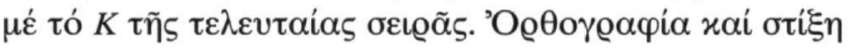

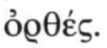

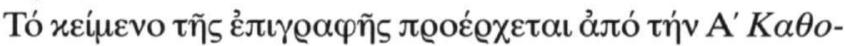

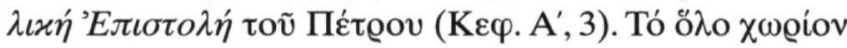

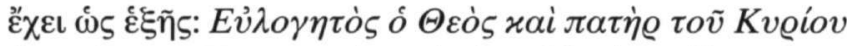

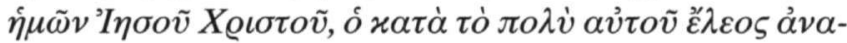

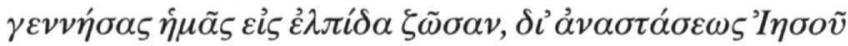

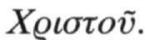

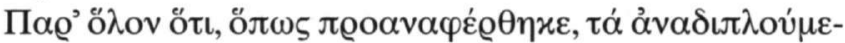

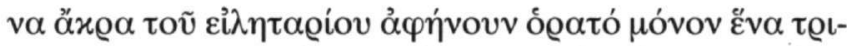

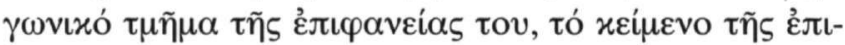

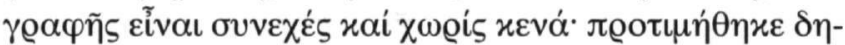

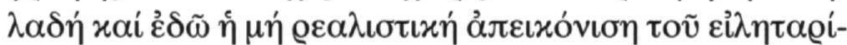




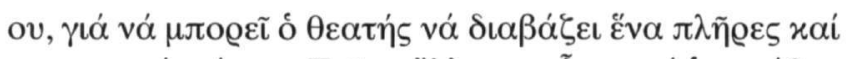

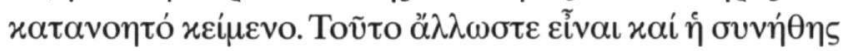

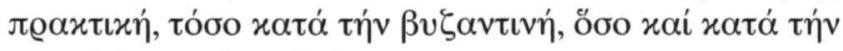
$\mu \varepsilon \tau \alpha \beta \vartheta \zeta \alpha v \tau \iota v \eta \dot{~ \pi \varepsilon \varrho i o \delta o . ~}$

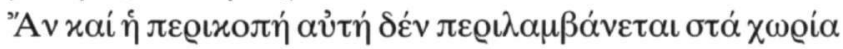

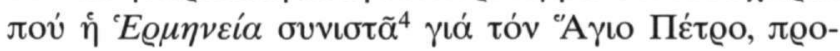

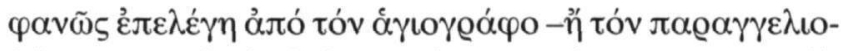

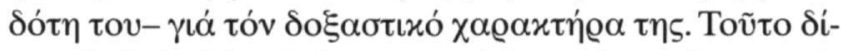

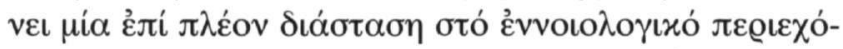

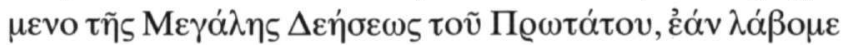

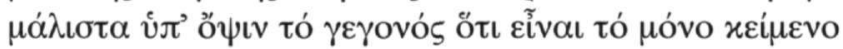

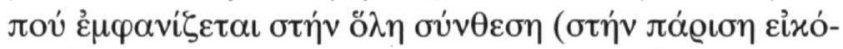

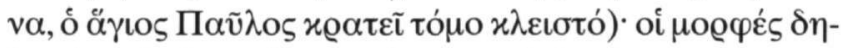

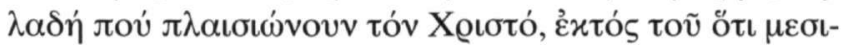

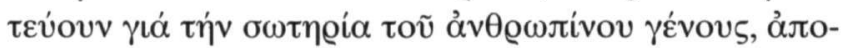

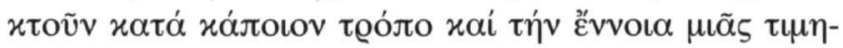

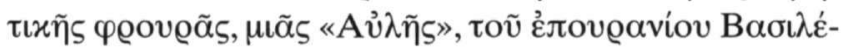

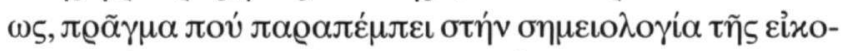

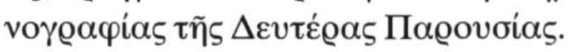

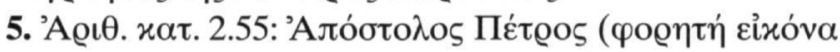

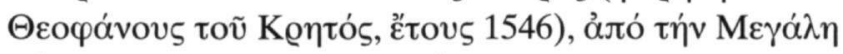

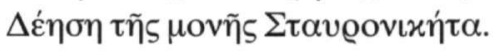

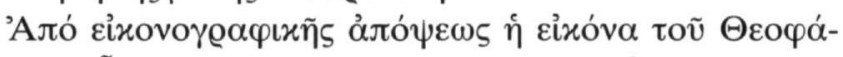

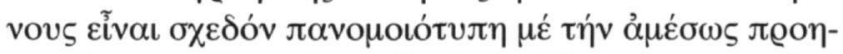

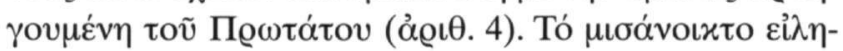

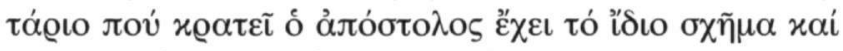

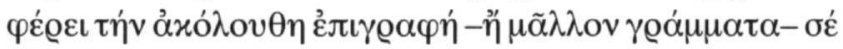

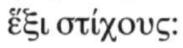

$$
\begin{aligned}
& \text {...] I ПАРАК[... } \\
& \text {...] KOY [... } \\
& \text {...]'АПЕ [... } \\
& \text {...] EIII [... } \\
& 5 \quad \ldots] N E[\ldots \\
& \text {...] OP [... }
\end{aligned}
$$

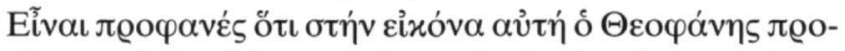

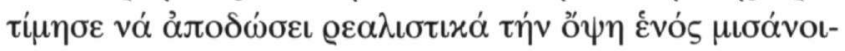

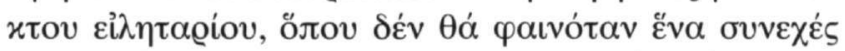

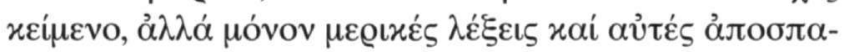
$\sigma \mu \alpha \tau i x \alpha \dot{~}$

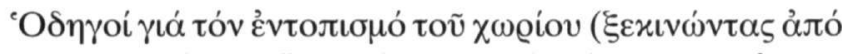

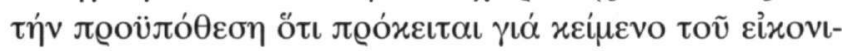

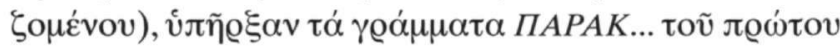

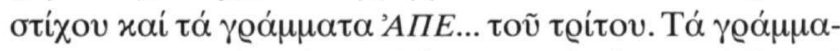

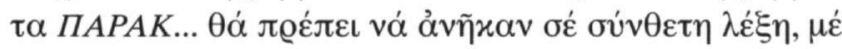

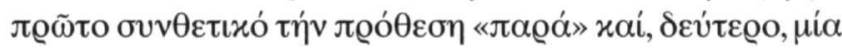

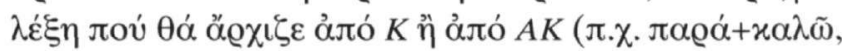

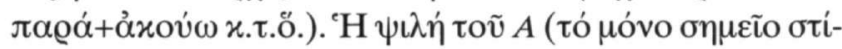

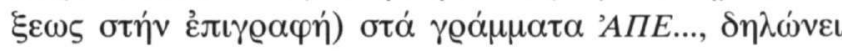

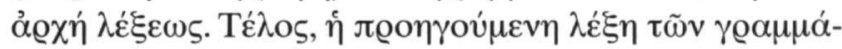

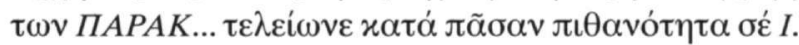

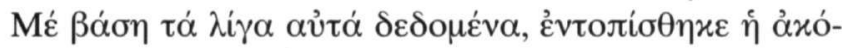

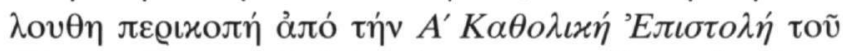

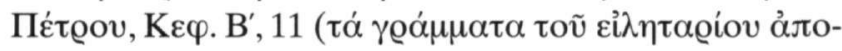

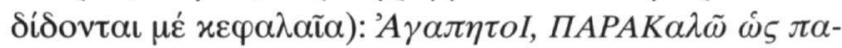

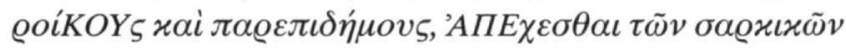

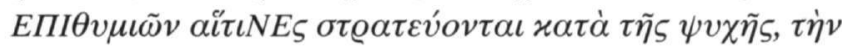

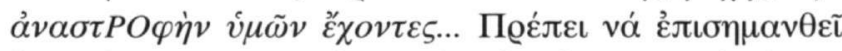

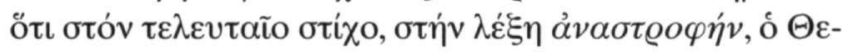

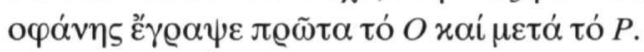

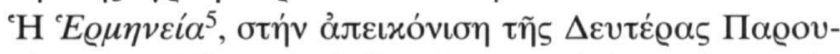

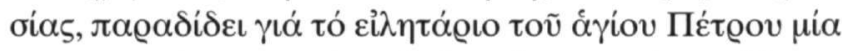

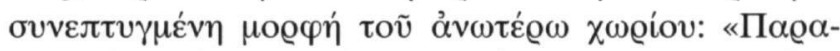

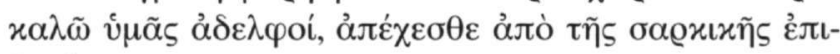
$\theta v \mu i \alpha \varsigma »$.

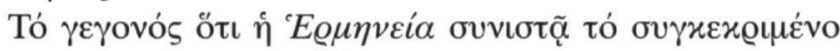

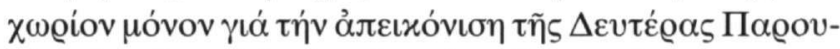

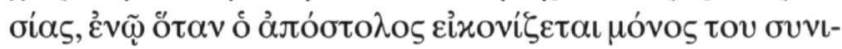

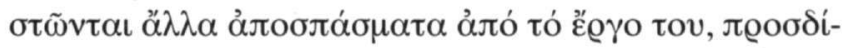

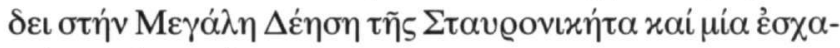

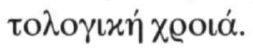

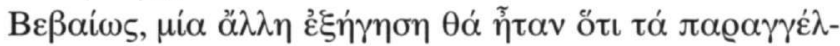

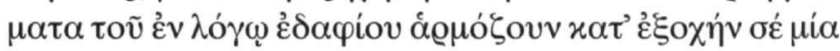

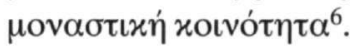

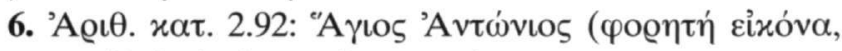

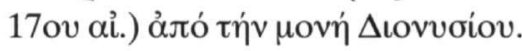

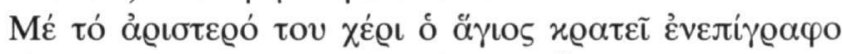

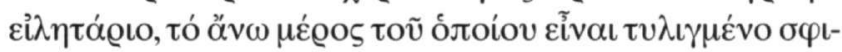

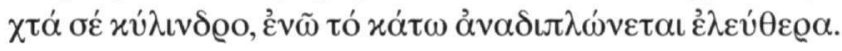

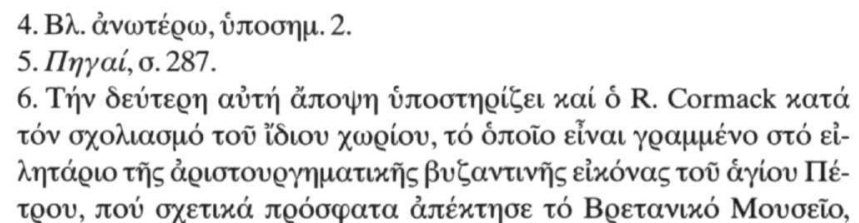

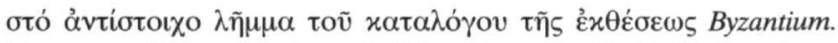
Treasures of Byzantine Art and Culture from British Collections, غ̇лц.

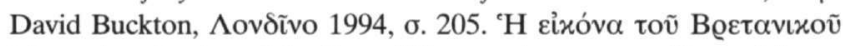

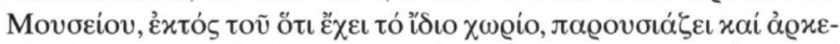

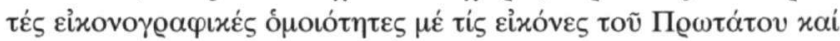

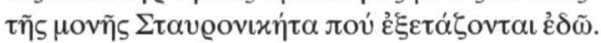




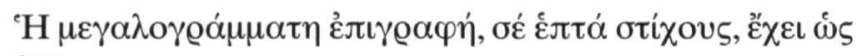
$\varepsilon \xi \tilde{\eta} \varsigma:$

\author{
${ }^{\prime} E X \Theta P \tilde{\Omega} N A C A P K \Omega N \triangle Y$ \\ CMEN $\Omega$ 'A $A A C T O ́ P \Omega N$ \\ KAIK KAKЕРГATSN ПAN \\ TEA $\tilde{Q} C T \Omega N \Pi P A K T E$ - \\ $5 \Omega N O Y ̉ \triangle E M I A B E \Lambda E M$

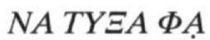 \\ PETPAC.
}

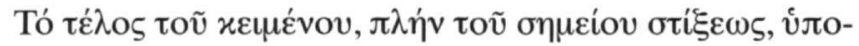

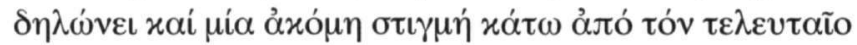

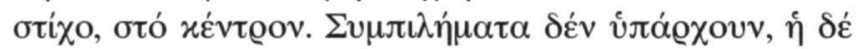

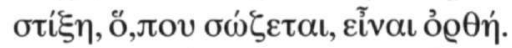

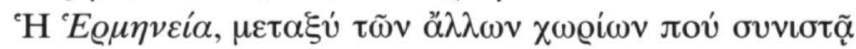

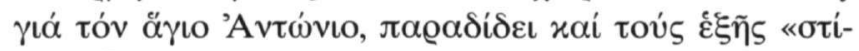

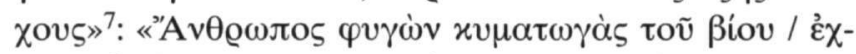

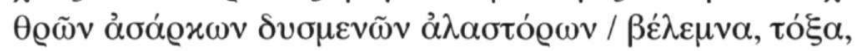

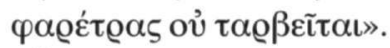

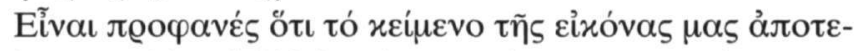

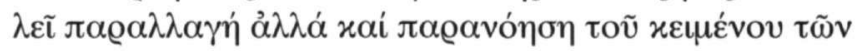

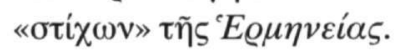

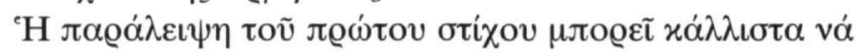

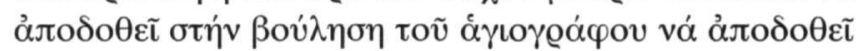

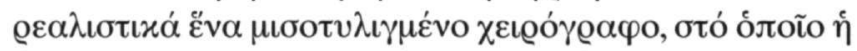

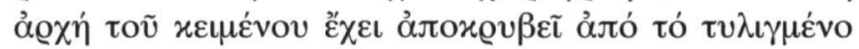

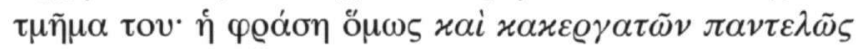

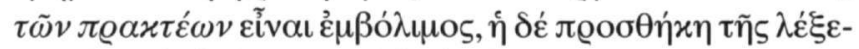

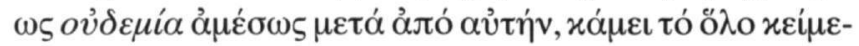

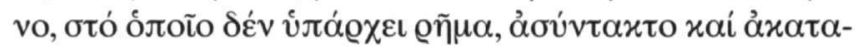

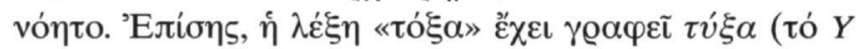

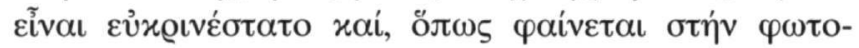

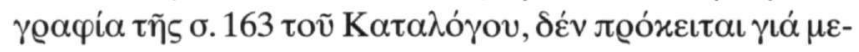

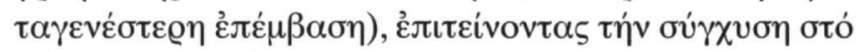

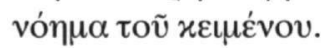

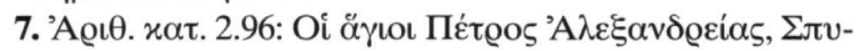

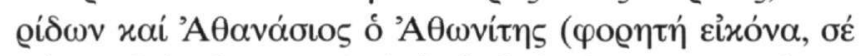

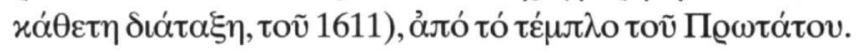

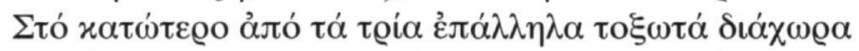

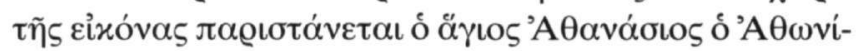

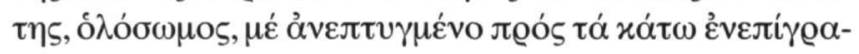

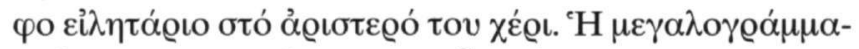

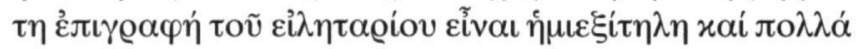

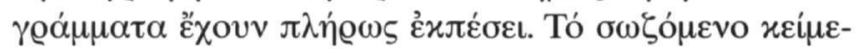

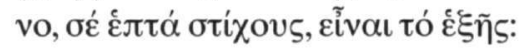

\author{
EN KA[...]PA \\ É $\Omega N[\ldots]$ \\ CET [...] \\ $K Y ́(P I O C), \Psi Y[\ldots]$ \\ $5 \triangle E$ TAPAX[...] \\ $\triangle I A B O ́ A[\ldots]$ \\ $K A \Theta E^{\prime}[\ldots]$
}

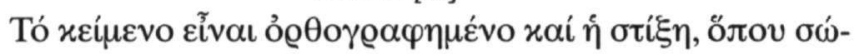

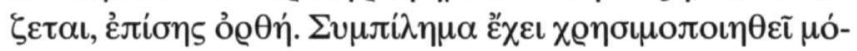

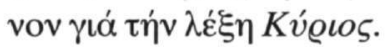

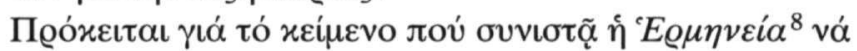

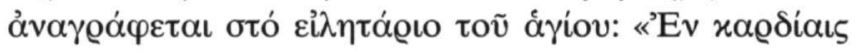

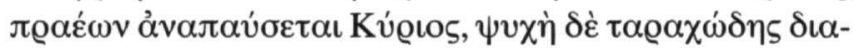

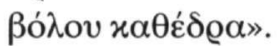

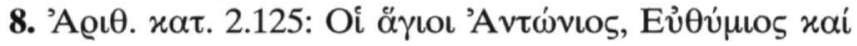

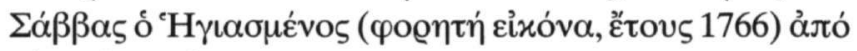

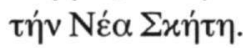

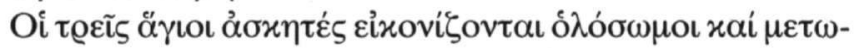

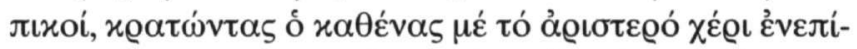

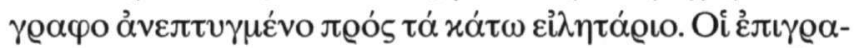

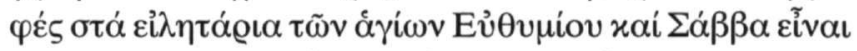

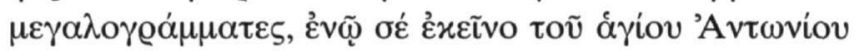

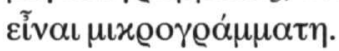

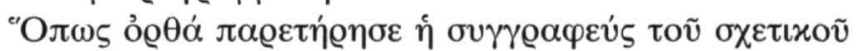

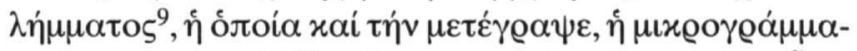

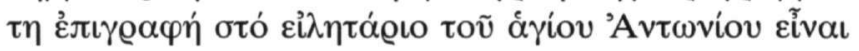

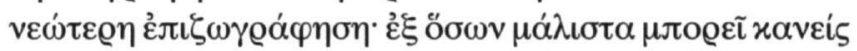

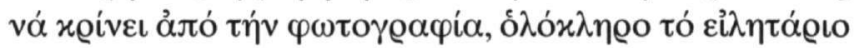

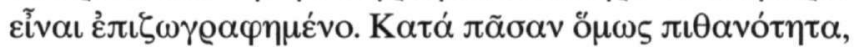

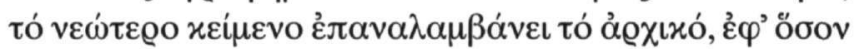

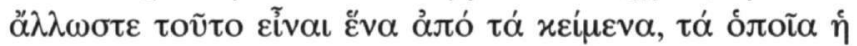

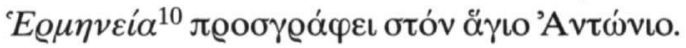

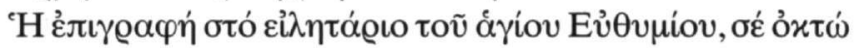

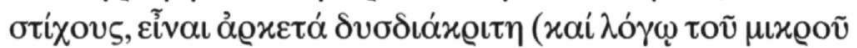

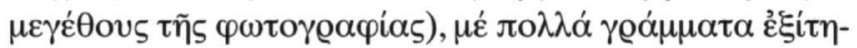

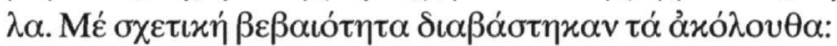

$$
\begin{aligned}
& \text { OI.... } \\
& \text { П.... } \\
& \triangle I A T P I B \\
& \text { ONTEC } \Phi \\
& 5 \text { IАAPГY } \\
& \text { PIAC Aイ } \\
& \text { TPIOI EIC }
\end{aligned}
$$

IN

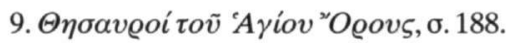

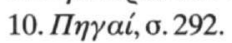




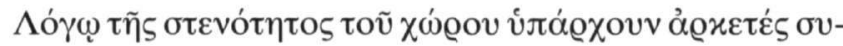

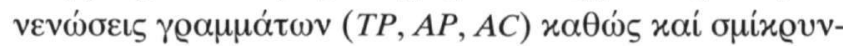

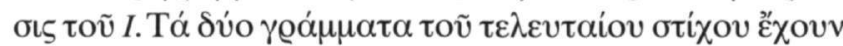

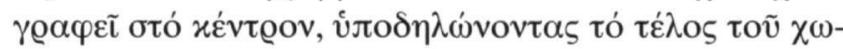

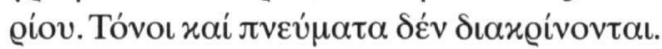

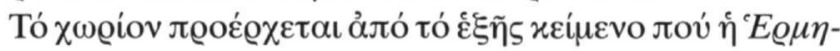

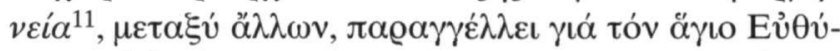

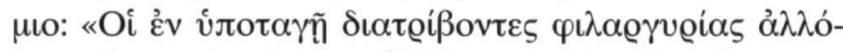

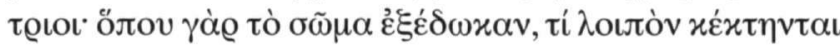
¿'oıv;».

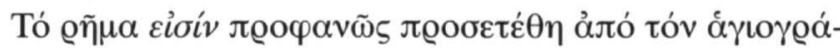

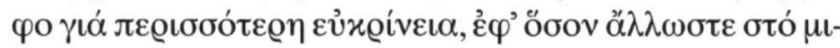

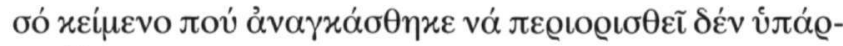

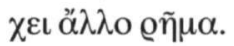

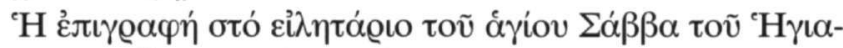

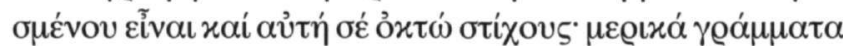

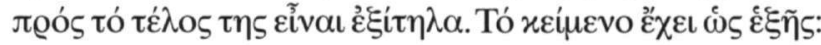

TEIXIC

ON TO KE

$\triangle \Lambda I O N C$

$O Y \tilde{\Omega} M O$

$5 N A X E$

KAI A A.O

KEA.ION

$M H$.....

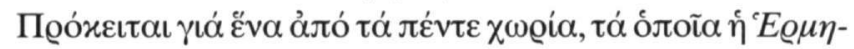

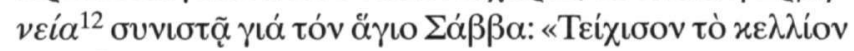

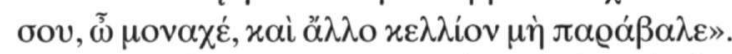

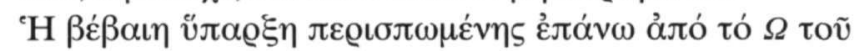

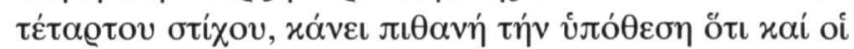

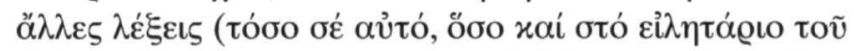

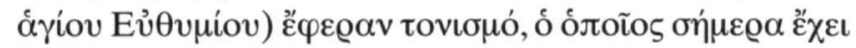

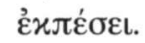




\section{Konstantinos Skabavias}

\section{NOTES DE LECTURE}

$\mathrm{U}$ n petit nombre d'icônes et de fresques représentant des saints tenant des rouleaux déployés, dont le texte n'a pas été transcrit et commenté dans le catalogue monumental de l'exposition «Trésors du Mont Athos» (2e édition, Thessalonique 1998), a fourni l'occasion à l'auteur de compléter ces quelques lacunes avec cet article. La lecture des textes a été basée sur les photographies du catalogue, dont on suit la numérotation.

1. Cat. No. 1.5 : Le prophète Ézéchiel (fresque détachée d'une coupole), du monastère de Pantocrator. Attribuée à Théophane le Crétois et datée entre 1535-1546.

Une grande partie du rouleau (à peu près la moitié supérieure et le tiers de la largeur) manque. Sur la partie conservée, on lit les lettres suivantes, en trois lignes: 'EII [.../ $\mathrm{K}(\mathrm{YPIO}) \mathrm{C} \cdot \mathrm{K}(\mathrm{AI})[\ldots / \mathrm{KÉ} \mathrm{ME}[\ldots$ Sur la quatrième ligne il y a un motif décoratif pour indiquer la fin du texte. Avec ces maigres indices, on a pu identifier l'inscription du rouleau

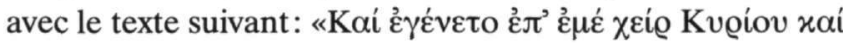

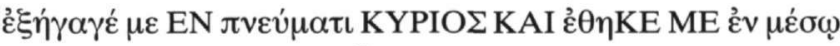

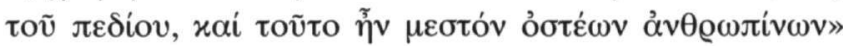
(Éz. XXXVII, 1). Les lettres conservées sont soulignées (: Et la main du Seigneur vint sur moi et le Seigneur m'a fait sortir en esprit et m'a posé au milieu du champ, et ceci était plein d'ossements humains...). Il est à noter que ce passage ne figure pas parmi ceux que le Manuel de peinture ${ }^{1}$ conseille pour Ézechiel.

2. Cat. No. 2.5 : Saint Pierre (icône portative du 12e s.), de Prôtaton.

La surface de læicône est assez endommagée et par conséquant, plusieures lettres de l'inscription du rouleau sont éffacées ou illisibles. On a pu établir le texte suivant:

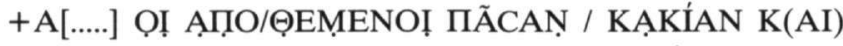

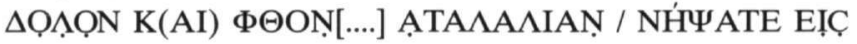

1. Denys de Fourna, Manuel d'iconographie chrétienne, accompagné de ses sources principales inédites et publié avec préface, pour la première fois en entier d'après son texte original, par A. Papadopoulo-Kerameus, aux frais de la Société Impériale Russe Archéologique, St. Petersbourg 1909.

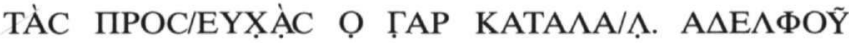

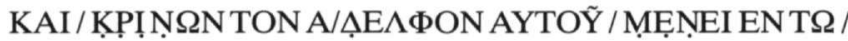
$\Theta A N A T \Omega+(:$ + Frères $(?)$, en rejettant toute méchanceté et fallace et médisance, revenez aux prières; car celui qui dit du mal pour son frère et juge son frère, reste dans la mort + ). Le texte ainsi établi est en réalité une compilation de quatre passages différents provenant de trois épitres apostoliques (Pierre A, II, 1 ; Pierre A, IV, 7 ; Jacques IV, 11-13 ; Jean A, III, 14). Le caractère admonitif du texte ainsi constitué est tout a fait approprié pour une communauté religieuse; ainsi, il ne serait pas très osé de supposer que l'icône en question était destinée dès le debut pour le lieu où elle se conserve jusqu'aujourd'hui.

3. Cat. No 2.14 : Saint jean Damascène (icône portative, débuts du 14e s.), de la Scète de St. Anne.

Bien que la plus grande partie de l'inscription est en belles lettres onciales, dans quelques cas le peintre a utilisé - par manque d'éspace - l'écriture minuscule; il y a aussi plusieures abbreviations. Le texte est le suivant: OI TH̃ $\Theta E O^{-}$

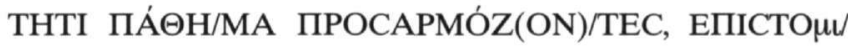
$\mathrm{ZE \sigma} \theta(\alpha \mathrm{\imath}) \Pi(\mathrm{AN}) \mathrm{T} \varepsilon \varsigma / \Lambda \mathrm{Q}[.$. (: Ceux qui attribuent à la divinité des passions, doivent tous être baillonés).

4. Cat. No. 2.52: Saint Pierre (icône portative de 1542), de la Grande Déèsis du Prôtaton.

L'apôtre tient le rouleau par le bas; la surface écrite est presque triangulaire. Le texte en lettres onciales disposées sur quatre lignes, est le suivant : EỴ̂ $\mathrm{K}(\mathrm{AI}) / \Pi(\mathrm{ATH}) \mathrm{P} / \mathrm{TOY}$ KYPIOY ( : Béni soit Dieu, le père du S[eigneur). Il provient de la première épitre catholique de St. Pierre (I, 3). Ce passage ne figure pas parmi ceux que le Manuel de peinture attribue à St. Pierre.

5. Cat. No. 2.55 : Saint Pierre (icône portative de Théophane le Crétois, datée 1546), de la Grande Déèsis du monastère de Stavronikita.

Du point de vue iconographique, cette icône est presque identique à la précédente. Théophane ayant préféré la façon réaliste pour représenter la surface écrite du rouleau, on ne distingue que les lettres suivantes, en six lignes: ПАРАK / KOY / 'AПE / EПI / NE / OP. Il s'agit du passage suivant, provenant de la première épitre catholique de saint Pierre (II,

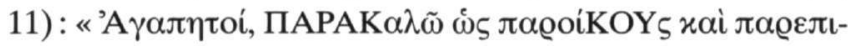




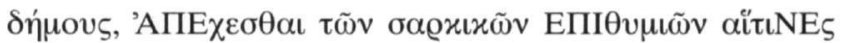

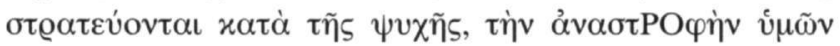

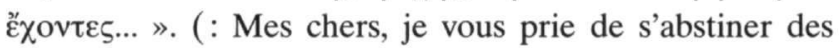
désirs charnels - comme si vous étiez des parèques et des étrangers de passage - lesquels assaillissent l'âme, ayant votre fréquentation...). Les lettres du rouleau sont rendues en majuscules. Une forme abrégée de ce passage est donnée par le Manuel... (p. 287) pour le rouleau de saint Pierre dans la composition de Jugement Dernier; ce qui conferrerait une nuance eschatologique à la Grande Déèsis de Stavronikita.

6. Cat. No. 2.92 : Saint Antoine (icône portative, 17e s.), du monastère de Dionysiou.

Le saint tient le parchemin par sa partie supérieure, enroulée comme un cylindre. Sur la partie déployée on lit:

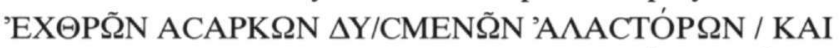

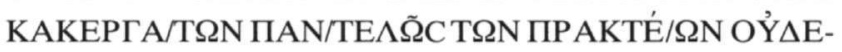
MIA BE $\Lambda E M / N A$ TY $\Xi A$ ФA.PETPAC. C'est une version incomplète et sur plusieurs points erronée d'un des passages

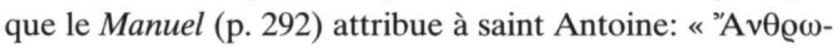

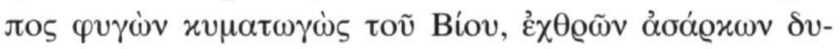

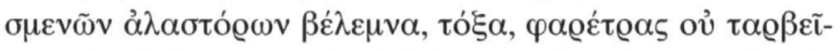
$\tau \alpha \iota$ » ( : Un homme qui a fuit les tempêtes de cette vie, n'a pas peur des flêches, arcs et carquois des ennemis incorporels et dévastateurs malveillants).

7. Cat. No. 2.96: Les saints Pierre d'Alexandrie, Spyridon et Athanase l'Athonite (icône portative), de l'iconostase de 1611 du Prôtaton.
Les saints sont disposés verticalement. Plusieures lettres du rouleau de saint Athanase sont détruites. On peut discerner le texte suivant: EN KA[...]PA / É $\Omega N[\ldots]$ / CET[...] /

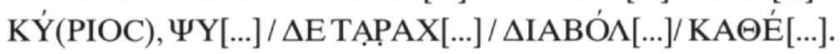
Il s'agit du passage que le Manuel (p. 295) attribue à ce saint:

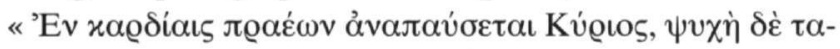

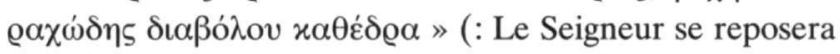
dans les âmes des gens bénins, tandis qu'une âme tumultueuse est le siège du diable).

8. Cat. No 2.125 : Les saints Antoine, Euthyme et Sabbas le bénit (icône portative, 1766), de la Nouvelle Scète.

Les inscriptions des rouleaux sont en lettres majuscules, à l'exception de celle de saint Antoine qui a été repeinte mais qui doit reproduire le texte original, vu que c'est celui que le Manuel attribue à saint Antoine. L'inscription du rouleau de saint Euthyme est assez endommagée; on a pu discerner le

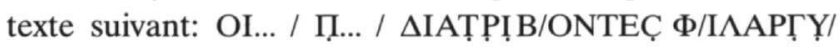
PIAC A $\Lambda \Lambda O$ /TPIOI EIC/IN. Il s'agit d'un des passages que le Manuel $^{2}$ recommande pour saint Euthyme : "Oi ع̉v v́jo-

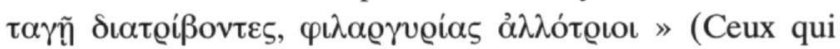
vivent en obéissance, sont étrangers à l'avarice). Le verbe EICIN (:sont), est un ajout du peintre. Le texte du rouleau de saint Sabbas est le suivant: TEIXIC/ON TO KE/A

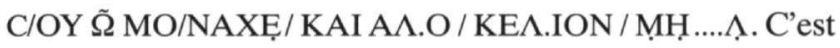
un des cinq passages que le Manuel (pp. 273 et 292) recom-

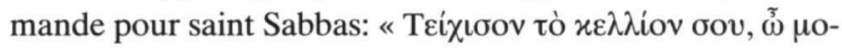

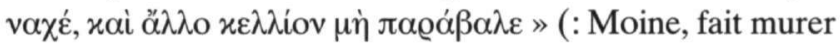
ta cellule et ne prends pas une autre). 